Europhys. Lett., 4 (6), pp. 657-662 (1987)

\title{
Dynamical Phase Transitions in 3-Dimensional Spin Glasses.
}

\author{
B. DERRIDA $\left({ }^{*}\right)$ and G. WeISBUCH $\left(^{* *}\right)$ \\ (*) SPT CEN-Saclay - 91191 Gif-sur-Yvette, France \\ (**) GPS ENS - 24 rue Lhomond, 75231 Paris Cedex 5, France
}

(received 27 April 1987; accepted in final form 11 June 1987)

PACS. 05.40. - Fluctuation phenomena, random processes, and Brownian motion.

\begin{abstract}
We study the time evolution of the distance between two configurations submitted to the same thermal noise for the $3 d \pm J$ Ising spin glass. We observe three temperature regimes: a high-temperature regime where the distances vanishes in the long-time limit. An intermediate-temperature regime where the distance has a nonzero limit independent of the initial distance. A low-temperature regime where the distance in the long time limit seems to depend upon the initial distance. For the sake of comparison, we have repeated our simulations for the ferromagnetic case.
\end{abstract}

Most of the physical properties of spin glasses which can be observed in the real world are dynamical effects due to slow relaxation processes (see ref. $[1,2]$ for a review). After a long debate about the existence or the nonexistence of a spin glass phase for 3-dimensional spin glasses, the most extensive numerical simulations [3] which have been done so far indicate that there is a spin glass phase for temperatures $T<T^{\mathrm{sg}} \simeq 1.2 \mathrm{~J}$ for the $\pm J$ model, but that slow relaxation effects appear in a large range of temperatures $T^{\mathrm{sg}}<T<T^{\mathrm{F}} \simeq 4.5 \mathrm{~J}$, where the data (spin autocorrelation function) can be fitted by stretched exponentials. Theoretical arguments [4] based on reasons similar to those which lead to Griffiths singularities [5] predict that below the critical temperature $T^{\mathrm{F}}$ of the ferromagnet, nonexponential decays should be observed.

In this letter, we present numerical data on the evolution of the distance $\langle D(t)\rangle$ between two configurations which are submitted to the same thermal noise, for the $3 d \pm J$ Ising spin glass on a cubic lattice. When measuring $\langle D(t)\rangle$ after a certain time (generally 500 iteration steps), we observe 3 regimes:

a high-temperature regime $T>T_{1}$ (with $T_{1} \simeq 4.1 \mathrm{~J}$ ) where, $\langle D(t)\rangle$ vanishes independently of the initial value $D(0)$;

an intermediate regime $T_{2}<T<T_{1}$ (with $T_{2} \simeq 1.8 \mathrm{~J}$ ) where, $\langle D(t)\rangle$ is nonzero and does not depend on the initial value $D(0)$;

a low-temperature regime $T<T_{2}$, where $\langle D(t)\rangle$ depends on $D(0)$. 
The simulations having been made for relatively short times $(t \leqslant 500)$, small systems $(N \leqslant 864)$, and few samples $(M \leqslant 800)$, our determination of $T_{1}$ and $T_{2}$ although not very accurate could be consistent with $T_{1}=T^{\mathrm{F}}$ and $T_{2}=T^{\mathrm{sg}}$.

The distance $D(t)$, in the limit $t \rightarrow \infty$, then appears as a useful order parameter for spin glasses, since it gives a clear signature of the intermediate phase $T_{2}<T<T_{1}$.

Methods. - Our numerical simulations are done for a system of $L^{3}$ Ising spins on a cubic lattice of linear dimension $L$ with periodic boundary conditions. The nearest-neighbour interactions $J_{i j}$ are randomly chosen

$$
\rho\left(J_{i j}\right)=\frac{1}{2} \delta\left(J_{i j}-J\right)+\frac{1}{2} \delta\left(J_{i j}+J\right) .
$$

The interactions $J_{i j}$ are quenched and symmetric $\left(J_{i j}=J_{j i}\right)$.

A spin configuration $\left\{S_{i}(t)\right\}$ evolves according to the following rule: at each time step $t$, all the local fields $h_{i}(t)$ are computed according to

$$
h_{i}(t)=\sum_{j} J_{i j} S_{j}(t)
$$

and the spins are then updated according to

$$
\begin{array}{lll}
S_{i}(t+1)=+1 \quad \text { with probability } & \frac{1}{1+\exp \left[-\frac{2 h_{i}(t)}{T}\right]}, \\
S_{i}(t+1)=-1 \quad \text { with probability } & \frac{1}{1+\exp \left[\frac{2 h_{i}(t)}{T}\right]},
\end{array}
$$

where $T$ is the temperature of the system. The dynamics are parallel dynamics. However, if we choose the linear size $L$ to be even, the system is decomposed into two independent sublattices which ignore each other and, therefore, stand as two different samples. One can easily check that dynamics (3) lead to the right thermal equilibrium for each sublattice in the long-time limit (i.e. the correlation functions between the spins of each sublattice averaged over time are the same as if they were computed at thermal equilibrium on the full lattice).

We consider two different initial configurations $\left\{S_{i}(0)\right\}$ and $\left\{S_{i}^{\prime}(0)\right\}$ at time $t=0$, and we let them evolve according to exactly the same rules: the $J_{i j}$ used to compute the fields $h_{i}(t)$ and $h_{i}^{\prime}(t)$ are the same and the random numbers used in (3) to decide whether $S_{i}$ and $S_{i}^{\prime}$ are + or -1 are the same (in particular if $h_{i}(t)=h_{i}^{\prime}(t)$, then $S_{i}(t+1)=S_{i}^{\prime}(t+1)$ ). A similar method was used recently to study the spreading of the damage caused by one spin flip [6]. We then measure the distance $D(t)$ between the two configurations as the number of spins which are different (i.e. such that $\left.S_{i}(t)=-S_{i}^{\prime}(t)\right)$

$$
D(t)=\frac{1}{4 N} \sum_{i=1}^{N}\left(S_{i}(t)-S_{i}^{\prime}(t)\right)^{2}
$$

The two sublattices are independent since at each time step they just exchange each other and for each cube of linear dimension $L$, we consider that we have two samples of size $N=L^{3} / 2$ spins.

In order to average $D(t)$ over disorder and thermal fluctuations, we repeat the simulations and generate $M$ samples by constructing $M / 2$ cubes. If two configurations 
become identical at time $t$, they remain identical at any later time. When we generate $M$ samples the first quantity we can measure is $M_{1}(t)$, the number of samples such that $\left\{S_{i}(t)\right\}$ and $\left\{S_{i}^{\prime}(t)\right\}$ are still different at time $t$ : one then defines a survival probability $P(t)$ by

$$
P(t)=\lim _{K \rightarrow \infty} \frac{M_{1}(t)}{M}
$$

(For any finite system at finite temperature $P(t)$ goes to 0 as the time goes to infinity. However, in the simulations described below, there is a large range of time when $P(t)$ remains almost constant. This behaviour is similar to the behaviour of the magnetization in finite systems.)

We then measure the average distance $\langle D(t)\rangle$ over those $M_{1}(t)$ samples which have survived and, therefore,

$$
D(t)=\lim _{M \rightarrow \infty} \frac{\sum_{s=1}^{M} D_{s}(t)}{M_{1}(t)},
$$

where $D_{s}(t)$ is the distance measured at time $t$ for the $s$-th sample.

In order to study how $\langle D(t)\rangle$ depends upon $D(0)$, three different sets of initial conditions were used: is then 1 .

A) Configuration $\left\{S_{i}(0)\right\}$ is random and configuration $\left\{S_{i}^{\prime}(0)\right\}=\left\{-S_{i}(0)\right\}$ for all $i . D(0)$

B) Configurations $\left\{S_{i}(0)\right\}$ and $\left\{S_{i}^{\prime}(0)\right\}$ are random and independent. $D(0)$ is then $1 / 2$.

C) Configuration $\left\{S_{i}(0)\right\}$ is random and configuration $\left\{S_{i}^{\prime}(0)\right\}$ is identical to $\left\{S_{i}(0)\right\}$ except for one spin on each sublattice. $D(0)$ is then $1 / N$.

The simulations have been performed for cubes of linear dimension $L=8$ (each sublattice having 256 spins) and $L=12$ (each sublattice having 864 spins). The results are averaged over 800 samples for $N=256$ and 200 samples for $N=864$. The numerical effort is thus roughly the same for the two sizes.

Spin glass results. - Figure 1 shows the survival probability $P(t)$ as a function of temperature $T$ for the three sets of initial conditions $A$ ), $B$ ) and $C$ ), after 500 time steps. Two regimes can be observed. Above $T_{1} \simeq 4.5 \mathrm{~J}, P(t)$ is 0 , whatever $D(0)$. Below $T_{1}$, we see in cases $A$ ) and $B$ ) a sharp increase of $P(t)$ up to 1 . Two different initial configurations never become identical. Even more surprisingly in case $C$ ), we see that two initial configurations which differ by a single spin have a probability of the order of 60 percent to remain different. The results do not seem to depend upon the size of the system, at least when we compare them for the cases $L=8$ and $L=12$. The results (not represented here) after 100 steps are very similar except for the transition region $T \simeq T_{1}$. Improving the quality of fig. 1 is not easy since the error bar decreases like $M^{-1 / 2}$, but does not decrease with the system size $N$.

Distances $\langle D(t)\rangle$ are plotted in fig. 2. They exhibit three different regimes.

For $T>T_{1},\langle D(t)\rangle$ vanishes for all three cases $\left.\left.A\right), B\right)$, and $\left.C\right)$.

In the range $T_{2}<T<T_{1}$ (with $T_{2} \simeq 1.8 \mathrm{~J}$ ), $\langle D(t)\rangle$ does not depend upon the set of initial conditions $A), B$ ) or $C$ ) or upon the system size; by comparing fig. $2 a$ ) and $b$ ), we see that $\langle D(t)\rangle$ has not evolved between times $t=100$ and $t=500$ and, therefore, seems to have already reached its long-time limit. 


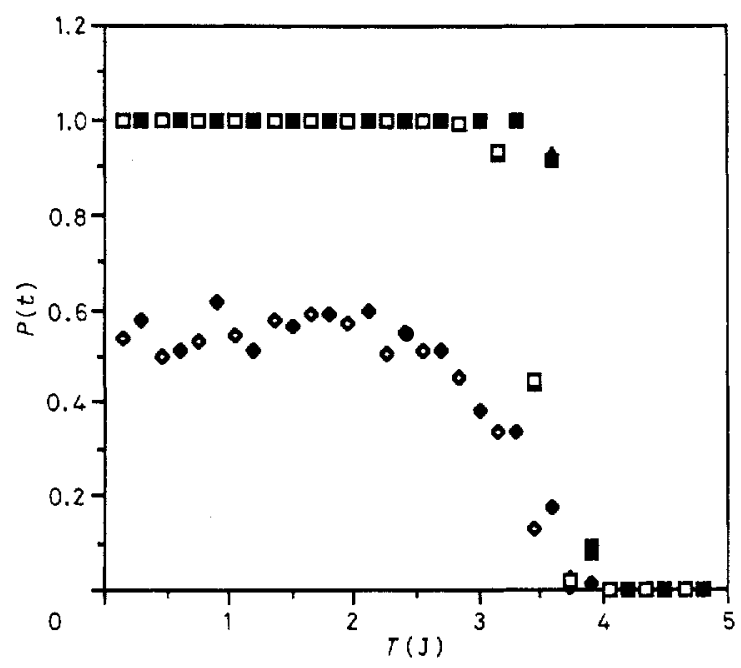

Fig. 1. $-3 d \pm J$ spin glass case. Survival probability, $P(t)$, that two initially different configurations remain different after $t$ times steps (here $t=500$ ), as a function of temperature $T$. The smoothness of the curves gives an idea of error bars which are not figured $(0.07$ for $L=12$ and 0.035 for $L=8)$. (White signs correspond to cubes of linear size $L=8$ and black signs to cubes of linear size $L=12$. Triangles are for initially opposed configurations, case $A$ ), squares for random configurations, case $B$ ), and diamonds for configurations differing initially by only 1 spin, case $C$ ). The triangles are masked by the squares when they coincide).

Lastly, in the range $T<T_{2}$, $\langle D(t)\rangle$ does depend upon $D(0)$. We see, however, that $\langle D(t)\rangle$ does not change with the system size $L$ and has very little change with time except in case $C$ ) where the difference between the two configurations takes a longer time to spread for the largest system $L=12$.

The existence of large range of temperatures where $\langle D(t)\rangle$ seems to have reached an equilibrium value independent of system size and initial conditions is in fact the main result of this paper. $T_{2}$ clearly depends upon iteration time and system size. One expects $T_{2}$ to
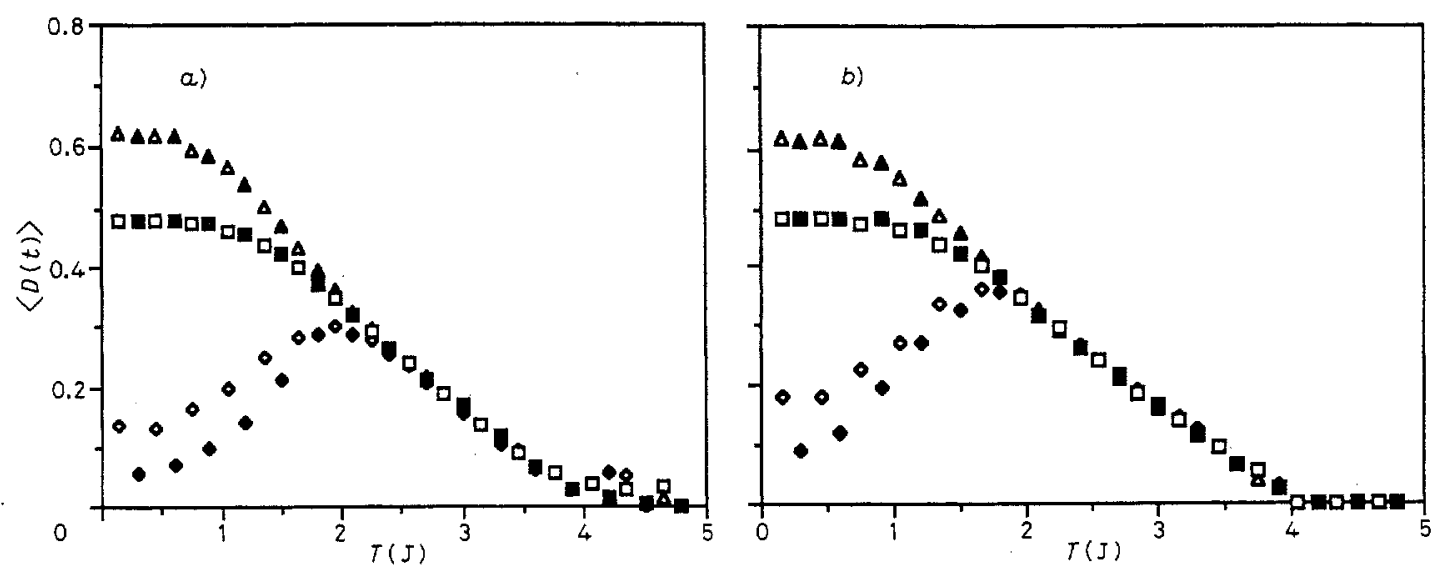

Fig. 2. - Spin glass case. Distance $\langle D(t)\rangle$ as a function of $T$. (The signs on the plots have the same meaning as in fig. 1.) $t=100$ for $a$ ) and 500 for $b$ ). In the temperature range between $2 \mathrm{~J}$ and $4 \mathrm{~J}$, the data for the three sets of initial conditions coincide. 
decrease with time, since when there is an equilibrium value of $\langle D(t)\rangle$, it takes longer to reach it at lower temperatures. On the other hand, $T_{2}$ should increase with $L$ because it is more difficult to eliminate the defects in larger systems. Further simulations would be needed in order to study the dependence of $T_{2}$ upon size $L$ and time $t$ in the limit when they both go to infinity. A precise determination of the upper temperature $T_{1}$ and of the critical behaviour of $\langle D(t)\rangle$ in its neighbourhood would also be of interest.

Ferromagnet. - In order to compare the above results with a better understood problem, we have repeated the above tests for the ferromagnetic problem with the same statistics, the same sizes, the same times and the same initial conditions. Figures 3 and 4 show our results after 500 time steps.

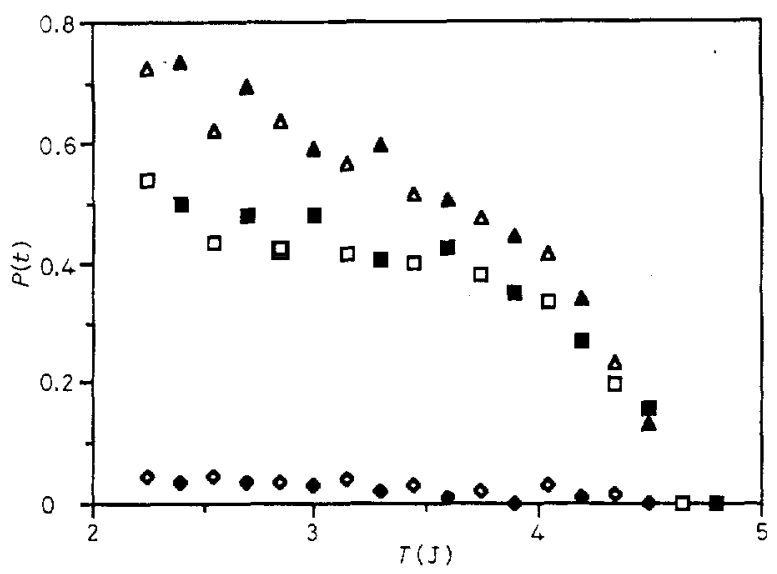

Fig. 3.

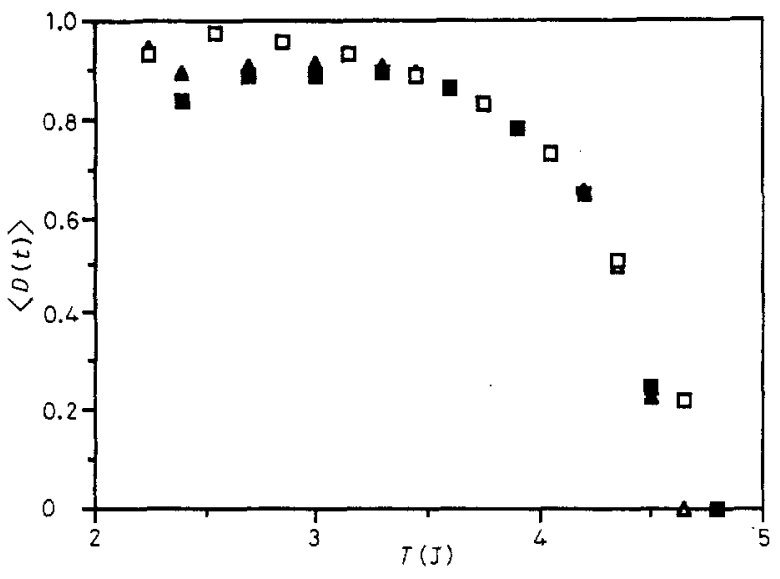

Fig. 4.

Fig. 3. - Ferromagnetic case. Survival probability, $P(t)$, that initially different configurations remain different after $t$ iteration steps (here $t=500$ ), as a function of temperature $T$. (The signs on the plots have the same meaning as in fig. 1).

Fig. 4. - Ferromagnetic case. Distance $\langle D(t)\rangle$ of initially distant configurations as a function of temperature $T$. time $=500$. (The signs on the plots have the same meaning as in fig. 1.)

We see that the survival probability depends now upon temperature for cases $A$ ) and $B$ ) and is very small (almost 0 ) for case $C$ ). This is because the initial configuration is random. A similar calculation with $S_{i}(0)=1$ for all $i$ 's, would give $P(t) \simeq 1$ for case $A$ ) and $P(t) \simeq 0.5$ for case $B$ ). Numerical data (not shown in the figures) indicate that $P(t)$ is still evolving at time $t=500$. $P(t)$ vanishes at a temperature $T_{1}$ close to the ferromagnetic-transition temperature $T^{\mathrm{F}}=4.5 \mathrm{~J}[3]$.

In fig. 4 we see the distance $\langle D(t)\rangle$ for cases $A$ ) and $B$ ) only. (The survival probability being so small for case $C),\langle D(t)\rangle$ would exhibit huge fluctuations due to the small number of samples.) The situation with respect to the relations between $\langle D(t)\rangle$ and $D(0)$ is very similar to the spin glass case.

For $T>T_{1} \simeq T^{\mathrm{F}},\langle D(t)\rangle$ vanishes in all cases.

In the range $T_{2}<T<T_{1},\langle D(t)\rangle$ has a limit independent of $D(0)$.

For $T<T_{2} \simeq 3.5 \mathrm{~J},\langle D(t)\rangle$ does depend upon $D(0)$.

For $T_{2}<T<T_{1}$, it is reasonable to say that $\langle D(t)\rangle$ is the distance between the + and the - phases and, therefore, that $\langle D(t)\rangle$ vanishes at $T=T_{\mathrm{c}}$ like the magnetization. One should 
notice that the temperature where $\langle D(t)\rangle$ vanishes agrees very well with the ferromagnetic transition temperature $T^{\mathrm{F}}$.

For $T<T_{2},\langle D(t)\rangle$ depends upon $D(0)$ because the system cannot eliminate the defects during time $t$.

The general picture is thus the same for spin glasses and ferromagnets, except for the survival probability and the shape of $\langle D(t)\rangle$ near $T_{1}$. After a long but finite time $t$, both systems exhibit three different dynamical regimes.

Conclusion. - A transition temperature in the long-time behaviour of $\langle D(t)\rangle$ has already been found in random nets of automata [7-10] and in nonsymmetric spin glasses [11]. An important result of this letter is to give evidence for an analogous transition for the $3 d \pm J$ Ising spin glass at $T_{1}$ (the higher transition temperature).

Further simulations are still needed to analyse the critical behaviour near $T_{1}$ and to see how $\langle D(t)\rangle$ depends upon time, dimension, magnetic field, the distribution of bonds, the symmetry of interactions, and the stochastic algorithm used to define the dynamics. It would also be interesting to establish relations between the dynamics of $\langle D(t)\rangle$ and that of physical quantities accessible to experiments.

It would also be interesting to do more simulations, in order to analyse the time and size dependence of $T_{2}$. In the ferromagnetic case, we expect $T_{2}$ to decrease with time $t$ and to increase with the system size $L$. Thus the limit of $T_{2}$ when $t \rightarrow \infty$ and $L \rightarrow \infty$ should depend on the way these limits are taken. For finite $L$ and $t$, the picture seems to be that for $T_{2}<T<T_{1}$, the system is either in the + or the - phase, whereas for $T<T_{2}$, the configurations still have defects. Displaying the configurations would be much useful to decide what are the defects which exist below $T_{2}$.

In the spin glass case, $T_{2}$ depends on time. Our simulation with 500 Monte Carlo steps gives $T_{2} \approx 1.8 \mathrm{~J}$ which is rather close to the spin glass transition temperature $\left(T^{\mathrm{sg}} \simeq 1.9 \mathrm{~J}\right)$ estimated from short-time Monte Carlo simulations [12]. It would then be very interesting to analyse the time dependence of $T_{2}$, in order to see whether it converges towards the transition temperature $T^{\mathrm{sg}}$ in the limit $t \rightarrow \infty$.

$$
* * *
$$

We acknowledge useful discussions with M. MÉzard and N. SourLas. We thank A. WEISBUCH for help in data processing.

\section{REFERENCES}

[1] Binder K. and Young A. P., Rev. Mod. Phys., 58 (1986) 801.

[2] Chowdhury D., Spin Glasses and other Frustrated Systems (World Scientific and Co.) 1986.

[3] OGielsky A., Phys. Rev. B, 32 (1985) 7384.

[4] Randeria M., Sethna J. P. and Palmer R. G., Phys. Rev. Lett., 54 (1985) 1321.

[5] Griffiths R. B., Phys. Rev. Lett., 23 (1969) 17.

[6] STAUFFER D., private communication.

[7] Derrida B. and Pomeau Y., Europhys. Lett., 1 (1986) 45.

[8] Derrida B. and Weisbuch G., J. Phys. (Paris), 47 (1986) 1297.

[9] DeRrida B. and StaUfFer D., Europhys. Lett., 2 (1986) 739.

[10] Weisbuch G. and Stauffer D., J. Phys. (Paris), 48 (1987) 11.

[11] Derrida B., to appear in J. Phys. Lett. A (1987).

[12] KIRKPATRICK S., in Ordering in Strongly Fluctuating Condensed Matter Systems, edited by T. RisTe (Plenum Press, New York, N.Y.) 1980, p. 459. 